'Departamento Cardiovascular. Hospital Clínico Universidad de Chile. Santiago, Chile. ${ }^{2}$ Carrera de Medicina. Facultad de Medicina. Universidad de Chile. Santiago, Chile. ${ }^{3}$ Laboratorio de Hemodinamia. Hospital Clínico Universidad de Chile. Santiago, Chile. Interno de Medicina. ${ }^{\text {b}}$ Tecnólogo Médico. 'Enfermera.

Recibido el 17 de mayo de 2017 , aceptado el 3 de octubre de 2017.

Correspondencia a: Dr. Héctor Ugalde Prieto ugaldehector@gmail.com

\section{Síndrome de tako-tsubo, caracterización clínica y evolución a un año plazo}

\author{
HÉCTOR UGALDE ${ }^{1}$, MARÍA CECILIA YUBINI 2,a, \\ MARÍA IGNACIA SANHUEZA ${ }^{2, a}$, FRANCISCO AYALA ${ }^{1}$, \\ ERNESTO CHAIGNEAU ${ }^{1}$, GASTÓN DUSSAILLANT ${ }^{1}$, SEBASTIÁN GARCÍA ${ }^{1}$, \\ ERIC FARÍAS ${ }^{3, b}$, KATIA VILLAGRA ${ }^{3, c}$, PAULA INOSTROZA ${ }^{3, c}$
}

\section{Tako-tsubo syndrome. Analysis of 37 cases}

Background: Tako-tsubo Syndrome (TTS) is characterized by transient regional systolic dysfunction of the left ventricle (LV), mimicking myocardial infarction. It accounts for 0.9-1.2\% of all acute coronary syndromes (ACS). Aim: To describe the incidence and characteristics of TTS within our population. Material and Methods: All patients diagnosed with ACS and TTS were selected from a clinical registry of all the coronary angiographies done in our hospital. Clinical features during initial presentation, hospital evolution and one year follow-up were analyzed. Results: The first case diagnosed in our hospital occurred in 2001. Since then, 4,433 coronary angiographies were done to patients with ACS until 2014 and 37 corresponded to TTS (0.83\% incidence). The mean age of patients was 64 years, $73 \%$ were female, and $62 \%$ had hypertension. All patients had an identifiable trigger factor, abnormal EKG and elevated troponin. The coronary angiography did not show lesions in 97\%. However, all had the characteristic extensive segmental-motility alteration with a mean ejection fraction of $44 \%$. All patients were treated initially as an ACS. Seven patients had complications, namely acute cardiac failure in six and stroke in one. No patient died. At one year of follow-up, 100\% showed normal segmental motility and ejection fraction, no patient had a new episode of TTS and all were alive. Conclusions: TTS is rare and the incidence found in this study is slightly lower than that reported elsewhere. TTS mimics ACS and it should be suspected by its clinical, electrocardiographic and enzymatic particularities. Coronary angiography helps to rule out other diagnosis. All patients normalize motility and ventricular function, which is the definitive differential feature respect to ACS.

(Rev Med Chile 2017; 145: 1268-1275)

Key words: Takotsubo Cardiomyopathy; Ventricular Dysfunction; Ventricular Dysfunction, Left; Acute Coronary Syndrome.
$\mathrm{E}$ síndrome de tako-tsubo (STT) o balonamiento apical transitorio del ventrículo izquierdo fue descrito en 1991 en población japonesa ${ }^{1}$. Se le denominó miocardiopatía símil tako-tsubo, nombre nacido de la similitud de la forma que adquiere el ventrículo izquierdo en la ventriculografía con una vasija utilizada por pescadores japoneses para pescar pulpos (Figura 1A). Consiste en la aparición brusca de aquinesia o hipoquinesia de los segmentos medios y apicales del ventrículo izquierdo, con hipercontractilidad basal (Figura 1B), asociado a un cuadro clínico similar a un síndrome coronario agudo (SCA). Suele ser gatillado por algún evento estresante y 


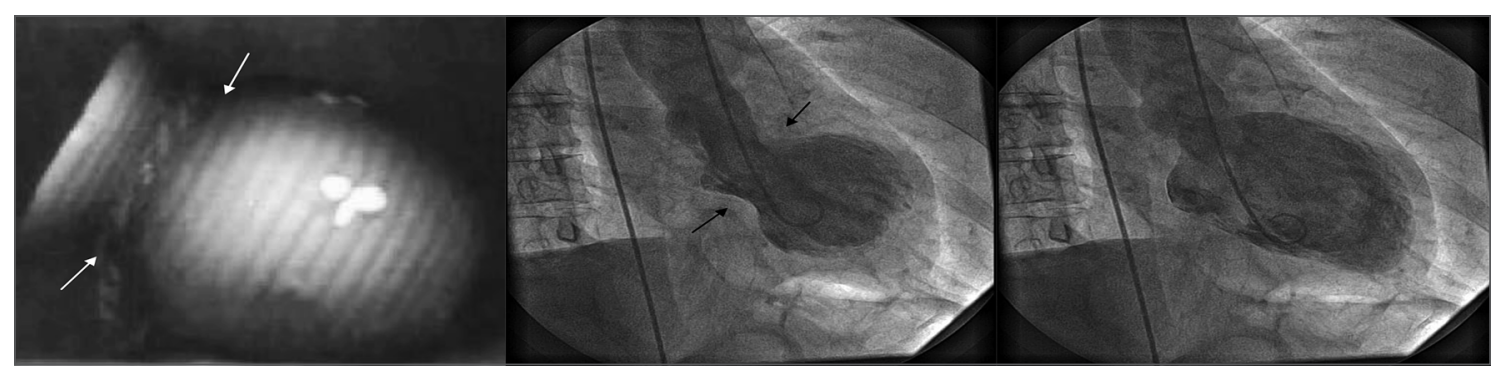

Figura 1. A: Vasija con la cual pescadores japoneses pescaban pulpos (TS), flechas muestran la zona similar a la ventriculografía con hipercontractilidad basal; B: Ventriculografía en sístole que muestra aquinesia anterior-apical-inferior, con hipercontractilidad basal (flechas); C: Ventriculografía en diástole.

característicamente se recupera en forma completa. Su causa es desconocida, se han planteado diferentes hipótesis, siendo la más aceptada actualmente, que correspondería a daño directo por catecolaminas ${ }^{2}$. Inicialmente fue descrito solo en población japonesa, hasta que unos años después fue descrita en otras locaciones ${ }^{3,4}$. Desde entonces múltiples estudios se han publicado, el mayor recientemente ${ }^{5}$, considerándose en la actualidad que entre 1 y $2,5 \%$ de los SCA son en realidad un STT ${ }^{6-8}$. En Chile solo se han publicado casos clínicos ${ }^{9,10}$, desconociéndose su incidencia y si nuestros pacientes $(\mathrm{P})$ se asemejan a lo descrito, lo cual motiva esta presentación, que pretende describir frecuencia de presentación, características clínicas, de laboratorio, angiográficas, evolución hospitalaria y a un año en P de nuestro país, así como comparar con series extranjeras.

\section{Método}

Desde un registro prospectivo de pacientes sometidos a coronariografía, seleccionamos todos los que presentaron un SCA desde enero de 2001 a diciembre de 2014, esto dado que el primer caso de STT diagnosticado por nosotros ocurrió el 2001. De ellos se seleccionaron retrospectivamente aquellos que cumplían los criterios diagnósticos modificados ${ }^{11}$ de la Clínica Mayo para STT. Revisamos sus características clínicas al ingreso, resultado de exámenes (en especial electrocardiograma [ECG], marcadores de daño miocárdico y ecocardiograma [ECO]), de la coronariografía, evolución hospitalaria y seguimiento clínico y ecocardiografico, comparándose sus características generales al ingreso y resultado de la coronariografía con el grupo de SCA del mismo período. Los resultados se muestran como promedios y desviación estándar o mediana y rango en caso de variables continuas y como porcentajes en variables categóricas. Las diferencias entre los grupos se analizan con el programa STATA, utilizando t de Student o $\chi^{2}$ con $\mathrm{p}<0,05$ como significativo.

\section{Resultados}

Cuatro mil cuatrocientos cuarenta y tres pacientes ingresan con diagnóstico de SCA en el período estudiado. De ellos, 37 P cumplían criterios para STT $(0,83 \%$ del total). Sus características generales las vemos en Tabla 1. Destaca el predominio de las mujeres, dentro de este, la mayoría sobre 50 años. De los factores de riesgo cardiovascular (FRCV), la hipertensión arterial (HTA) fue el más frecuente, seguido por diabetes mellitus (DM) y tabaquismo. Al comparar con el grupo SCA, en este la edad era menor, predominaban los hombres, y en las mujeres eran igualmente más frecuentes las pacientes sobre 50 años. En relación a FRCV, igualmente fue la HTA el principal, seguido por tabaquismo y dislipidemia, sin diferencias significativas con el STT.

En todos nuestros pacientes con STT se identificó un elemento estresante previo al inicio de sus síntomas (Tabla 2), en $11 \mathrm{P}(29,7 \%)$ este fue reconocido posterior al diagnóstico. Destaca un mayor porcentaje de elementos de tipo físico (estrés corporal principal c/s componente psicológico), casi $60 \%$ del total, los más frecuentes fueron los asociados a alguna cirugía y a infeccio- 
Tabla 1. Características generales al ingreso

\begin{tabular}{|c|c|c|c|c|c|}
\hline & STS & $\%$ & SCA & $\%$ & $\mathbf{p}$ \\
\hline Número & 37 & 8,3 & 4.406 & 92,7 & \\
\hline Edad & $\begin{array}{c}64,2 \pm 12,5 \\
(34-87)\end{array}$ & & $\begin{array}{c}59,4 \pm 12,7 \\
(15-100)\end{array}$ & & 0,02 \\
\hline Sexo femenino (SF) & 28 & 75,7 & 1.116 & 25,3 & $<0,01$ \\
\hline Edad SF & $\begin{array}{c}65,1 \pm 12,3 \\
(42-87)\end{array}$ & & $\begin{array}{c}63,8 \pm 11,8 \\
(25-95)\end{array}$ & & 0,5 \\
\hline$S F \geq 50$ años & 24 & 85,7 & 984 & 88,2 & 0,91 \\
\hline Edad SM & $\begin{array}{c}61,6 \pm 13,5 \\
(34-77)\end{array}$ & & $\begin{array}{c}57,9 \pm 11,7 \\
(15-100)\end{array}$ & & 0,06 \\
\hline HTA & 23 & 62,2 & 2.464 & 55,9 & 0,55 \\
\hline DM & 8 & 21,6 & 947 & 21,5 & 0,86 \\
\hline Tabaquismo & 8 & 21,6 & 1.423 & 32,3 & 0,23 \\
\hline Dislipidemia & 7 & 18,9 & 1.243 & 28,2 & 0,28 \\
\hline
\end{tabular}

$p<0,05$ : significativo.

\section{Tabla 2. Factores asociados al inicio del cuadro}

\begin{tabular}{|lrc|}
\hline Factores asociados & n & \% \\
\hline Estrés emocional & 15 & 40,5 \\
Problema familiar & 10 & 66,6 \\
$\quad$ Fallecimiento cercano & 5 & \\
$\quad$ Discusión con hijo-marido & 3 & \\
Enfermedad severa marido & 2 & \\
\hline Estrés laboral agudo & 4 & 26,7 \\
$\quad$ Discusión con jefe & 3 & \\
Despido & 1 & \\
Asalto vía publica & 1 & 6,7 \\
\hline Estrés físico & 22 & 59,5 \\
\hline Esfuerzo físico mayor & 3 & 13,6 \\
\hline Sepsis, diferentes focos & 4 & 18,2 \\
\hline Cirugía reciente (< 12 h) & 4 & 18,2 \\
\hline Complicación en inducción anestésica & 2 & 9,1 \\
\hline Dolor intenso-prolongado & 3 & 13,6 \\
\hline Otros eventos de salud: & 6 & 27,3 \\
$\quad$ Obstrucción bronquial severa, sincope, & & \\
$\quad$ accidente Isquémico transitorio, conexión & & \\
$\quad$ a ventilación mecánica, crisis vertiginosa & & \\
severa, complicación de endoscopia & & \\
\hline
\end{tabular}

nes sistémicas graves, entre los de tipo emocional (estrés psicológico) predominaron los de tipo familiar, especialmente fallecimiento de familiar cercano. Al comparar según sexo se observó una distribución similar, predominando los estresores físicos, $60,7 \%$ en mujeres y $56,6 \%$ en hombres.
Los síntomas que motivaron la consulta fueron dolor torácico de tipo anginoso en $29 \mathrm{P}$ $(78,4 \%)$, disnea aguda en $7 \mathrm{P}(18,9 \%)$ y en $1 \mathrm{P}$ síncope $(2,7 \%)$. En todos se efectuó de inmediato ECG, 15 P presentaron supradesnivel ST (SDST) $(40,5 \%), 11 \mathrm{P}$ alteraciones en la onda $\mathrm{t}(29,7 \%)(\mathrm{t}$ invertida sugerente de isquemia), en $9 \mathrm{P}$ fue normal $(24,3 \%), 1 \mathrm{P}$ con marcapaso definitivo y otro con bloqueo completo de rama izquierda. La mediana del QTc fue $0,48(0,28-0,65)$, en 29 P estaba prolongado $(78,4 \%)$. En la evolución todos desarrollaron ondas $t$ negativas anchas y profundas entre 5-10 derivaciones (mediana de 8), siempre comprometiendo de V2-V6 (Figura 2, ECG 2). La troponina I inicial estuvo alterada en $36 \mathrm{P}(97,3 \%)$ no así la CKMB que fue elevada en 18 de 34 P (52,9\%). En la evolución, todos elevaron troponina, valor máximo fue una mediana de $2,4 \mathrm{ng} / \mathrm{ml}(0,3-23)$, $11 \mathrm{P}(29,4 \%)$ superan los $4 \mathrm{ng} / \mathrm{ml}$.

Fueron ingresados con diagnóstico presuntivo de SCA, sea infarto agudo al miocardio (IAM) $20 \mathrm{P}(54,1 \%)$, angina inestable $16 \mathrm{P}(43,2 \%)$ o insuficiencia cardiaca aguda y probable IAM en el restante. Treinta y un pacientes presentaban hemodinamia estable y sin signos de falla cardiaca $(83,8 \%), 4 \mathrm{P}$ con edema pulmonar $(10,8 \%), 2 \mathrm{P}$ en shock cardiogénico (SC) (5,4\%), 3 P requirieron conexión a ventilación mecánica y $1 \mathrm{P}$ balón de contrapulsación aórtico. Ninguno presentó arritmias ventriculares sostenidas, así como tampoco arritmias supraventriculares prolongadas. 


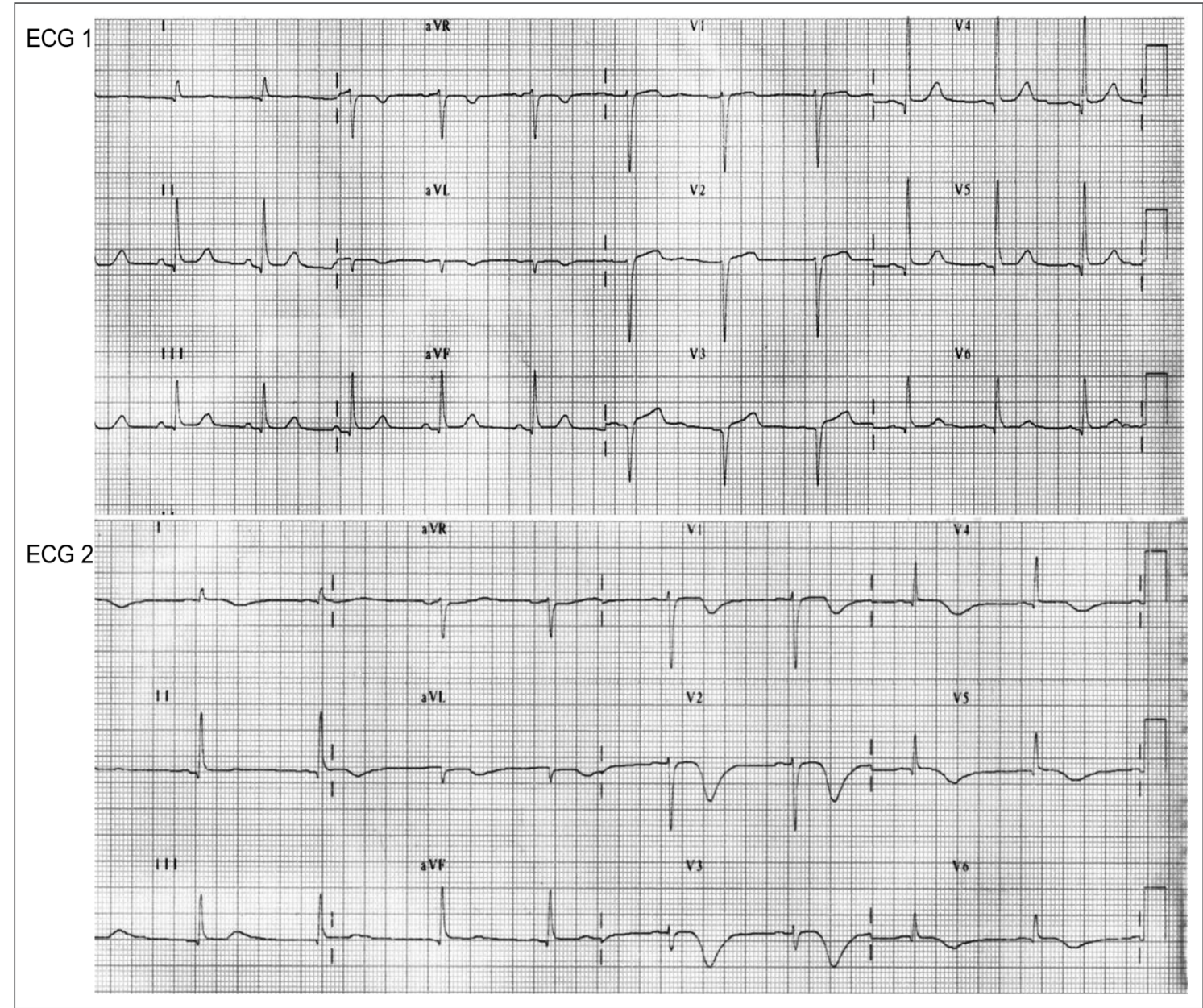

Figura 2. ECG 1: ECG inicial que muestra leve ascenso ST anteroseptal con QS en V3; ECG 2: al día siguiente, muestra aparición de $\mathrm{T}(-)$ ancha en 8 derivaciones y recuperación de $\mathrm{R}$ en $\mathrm{V} 3$.

En el tratamiento farmacológico, todos recibieron aspirina, $19 \mathrm{P}$ heparina $(51,4 \%), 18 \mathrm{P}$ nitroglicerina endovenosa $(48,6 \%), 14 \mathrm{P} \beta$-bloqueo $(37,8 \%)$ y $6 \mathrm{P}$ inótropos positivos (16,2\%). En $17 \mathrm{P}$ $(45,9 \%)$ se efectuó inmediatamente coronariografía; $1 \mathrm{P}$ con SDST recibió trombolisis endovenosa (el pabellón de angiografía estaba en reparación), en este paciente y en otros $9 \mathrm{P}$ la coronariografía fue efectuada al día siguiente, en los restantes, al tercer día; $36 \mathrm{P}$ no tenían lesiones coronarias significativas (97,3\%), $27 \mathrm{P}$ sin lesiones (73\%) (Figura 3), 9 P lesiones leves ( $<50 \%$ obstrucción) y solo $1 \mathrm{P}$ con lesión de $50 \%$ de aspecto estable en arteria descendente anterior distal. Entre los pacientes con SCA del mismo período, 7,9\% no tuvieron lesiones coronarias y $81,8 \%$ de ellos tuvieron al menos un vaso con lesión severa. Todos tuvieron alteraciones de motilidad segmentaria tipo tako-tsubo, fracción de eyección (FE) promedio de 44,3\%, $23 \mathrm{P}$ con $\mathrm{FE}<50 \%(62,2 \%)$.

En la evolución, $1 \mathrm{P}$ presentó el día 3 un accidente vascular encefálico de tipo isquémico, tratado con trombolisis, con recuperación completa posterior. La evolución en todos fue hacia la mejoría, fueron seguidos con ECO, todos presentaron mejoría de la motilidad y $\mathrm{FE}$ a los 3 días de evolución, 12 P normalizan la ECO antes de los 14 días (37,8\%), $29 \mathrm{P}$ antes del mes $(78,4 \%)$ y todos antes de los 3 meses. A 4 P se les efectuó resonancia nuclear magnética cardiaca por duda 


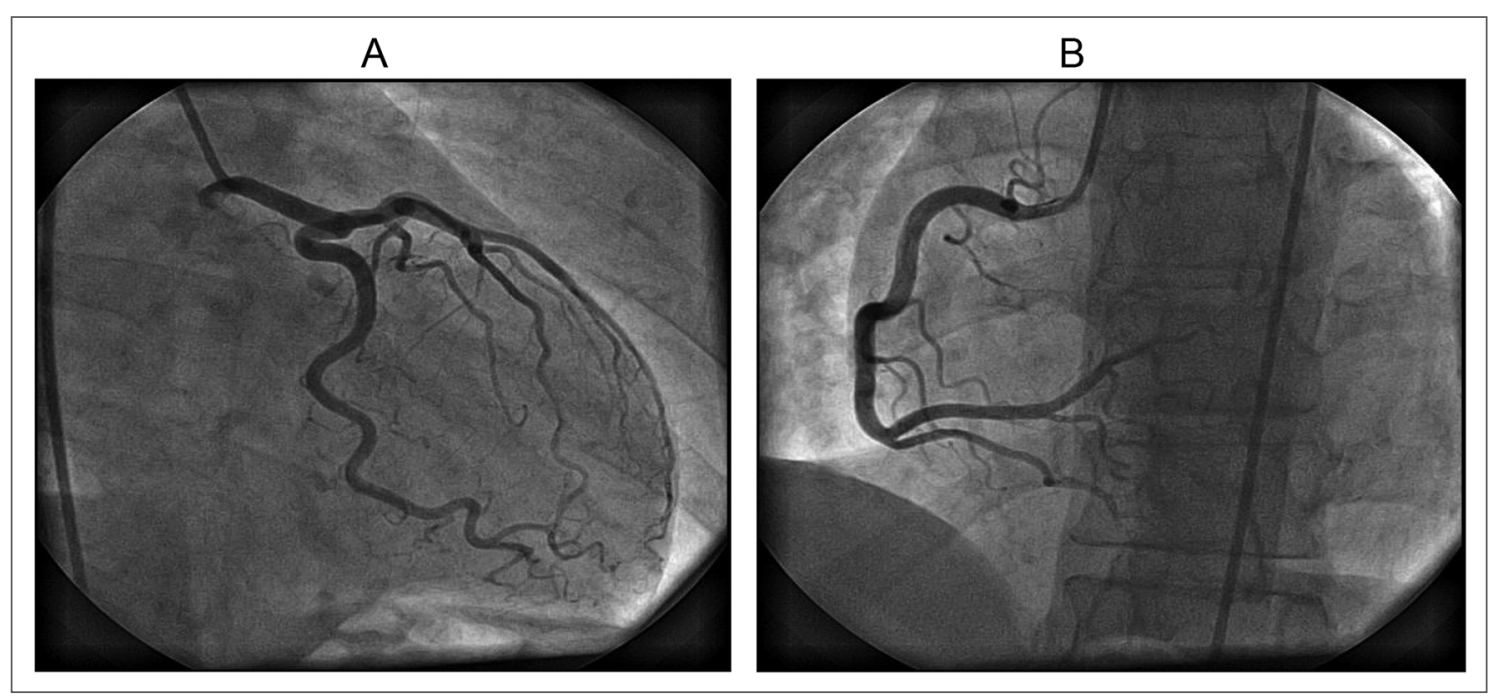

Figura 3. A: Coronariografía izquierda, con tronco común, descendente anterior y circunfleja sin lesiones; B: Arteria coronaria derecha, sin lesiones.

diagnóstica, descartándose miocarditis o IAM. Egresaron en promedio el día 10, no hubo fallecidos durante la hospitalización; $95 \%$ egresó con aspirina, $87 \%$ con $\beta$-bloqueo, $73 \%$ con estatinas y $57 \%$ con inhibidores de enzima de conversión (IECA). Al seguimiento, al menos por 1 año, no hubo nuevos eventos, nuevas hospitalizaciones ni fallecidos.

\section{Discusión}

Este registro de STT es el primero descrito en nuestro país, entidad descrita a principios de la década 1990-99 en Japón ${ }^{1}$ y posteriormente en otras locaciones $^{3,4}$, sin encontrar, hasta ahora, series en población latinoamericana, solo casos aislados ${ }^{12}$, los que aún se siguen publicando en distintos lugares del mundo. La publicación reciente del registro multinacional pretende dar una respuesta global a esta patología ${ }^{5}$, dado el alto número de casos incluidos, pero creemos que esto aún no se ha logrado del todo. Nuestro objetivo fue mostrar nuestra experiencia en esta patología, aún no bien comprendida.

La incidencia obtenida por nosotros fue de $0,83 \%$, algo menor a lo mostrado por diferentes series del mundo ${ }^{13}$, pero cabe hacer notar que solo incluimos casos de STT de la forma apical u originalmente descrita, lo que no ocurre en varias series que consideran las otras variedades descritas de miocardiopatía de estrés, como la medio ventricular, basal y focal, las que en el registro internacional corresponden casi a $20 \%$ del total ${ }^{5}$. En nuestro grupo no tenemos casos descritos de estas variedades, lo cual puede ocurrir porque en realidad no hemos tenido estos casos o por falta de diagnóstico, como así debe haber ocurrido antes del año 2001 con el STT. En relación a igualar el STT clásico con las otras variedades, no nos parece tan claro que correspondan a la misma entidad, aunque no podemos afirmar lo contrario, creemos que aún falta mucho por dilucidar para asegurar este postulado como una certeza.

Nuestros pacientes corresponden a lo clásicamente descrito, predominio marcado de mujeres $^{5,8,11,13-15}$, totalmente opuesto al SCA. El por qué ocurre no está claro. Se postula que los hombres en su evolución natural han sido sometidos a mayores eventos estresantes de tipo físico con lo que pudieran haber desarrollado mayor resistencia al efecto catecolaminérgico. Se ha demostrado que la densidad de receptores adrenérgicos en la membrana de los cardiomiocitos es mayor en los hombres y se postula que su saturación podría llevar más tiempo determinando menor riesgo a eventos adversos a catecolaminas ${ }^{16}$. Edad promedio de 65 años, similar a los demás registros ${ }^{5,13}$ y levemente superior 
en STT que SCA, básicamente dependiente de la mayor edad de las mujeres en ambas patologías, siendo en ambos casos, la mayoría de las pacientes mayores de 50 años, también similar a lo ya descrito $^{5,13}$. En los trabajos publicados se destaca que esta patología es predominantemente presente en mujeres posmenopáusicas, como característica diferencial, pero esto también ocurre en las mujeres con SCA, la mayoría también pertenecen al grupo postmenopáusico, lo que en realidad, hace difícil plantear este argumento de diferenciación. Los FRCV también son mencionados por algunos como un factor diferenciador, mencionándose como menores en el grupo de $\mathrm{STT}^{13}$, en particular una menor incidencia de diabetes mellitus ${ }^{17,18}$. En nuestro trabajo vemos que el FRCV predominante es HTA, tal ocurre en la mayoría de los trabajos publicados ${ }^{13}$, incluso superior a los $\mathrm{P}$ con SCA, siendo la DM similar en ambos grupos; tabaquismo y dislipidemia tuvieron una incidencia menor, no obstante, no podemos asumir estos datos como un elemento diferenciador relevante al momento del diagnóstico inicial, y esto queda de manifiesto al revisar las diferentes series, todas con diferencias ${ }^{13}$.

En todos nuestros pacientes existió un elemento estresante previo, predominando estresores de tipo físico, coincidente con la mayoría de descripciones ya publicadas ${ }^{5,13,17,19}$. No todas las publicaciones describen un elemento estresante previo, la mayoría de ellas encuentra un grupo en que no se identifica estresante $(20-30 \%)^{5,13,15}$, elemento que está incluido como requisito menor en algunos de los diferentes criterios para diagnóstico de STT ${ }^{20}$. Esto podría explicarse por una inadecuada historia clínica, considerando que la mayoría de los estudios es retrospectivo, también por inclusión de casos que no son realmente STT, así como también la posibilidad de que existan casos en que el factor estresante no logre ser reconocido. Entre los factores estresantes no se aprecian diferencias significativas en el tipo de factor predominante según sexo, aunque en algún estudio mencionan como más prevalente el factor físico en hombres y el emocional en mujeres ${ }^{15}$.

La sintomatología inicial en nuestros pacientes fue similar a lo descrito, siendo el dolor torácico el síntoma más relevante seguido de la disnea aguda $^{5,13,18,19,20}$. El ECG inicial en 70\% de nuestros pacientes estaba alterado, siendo el SDST la alteración más frecuente, concordante con lo publicado en la literatura ${ }^{13,17-19}$. Igualmente, la mayoría de los P presentaron un QTc prolongado, hecho frecuente, pero variable en su incidencia ${ }^{13}$. En todos los pacientes, el registro de EGC posterior al evento inicial desarrolló una $t$ negativa ancha y profunda, alteración característica del STT, que ocurre durante la evolución del síndrome, un porcentaje menor la tiene desde el ingreso ${ }^{13,18,19}$ y la mayor parte la desarrolla posteriormente ${ }^{18,19,21-23}$. Se ha intentado diferenciar electrocardiográficamente al STT del IAM ${ }^{24}$, definiéndose criterios para aquello, pero en la realidad, la diferencia se obtiene principalmente con la evolución y aún no se podría pretender plantear el diagnóstico inicial de STT en un paciente con dolor torácico o disnea y ECG alterado, pues por simple lógica, la probabilidad de que tenga un STT y no un SCA, dada la incidencia de ambos, es muy baja y el manejo inicial del SCA, en especial de SCA con SDST no debe esperar la evolución clínica posterior que confirme un STT. La realización de coronariografía precoz nos dará la posibilidad cierta de plantear el diagnóstico de STT, pero también realizar el tratamiento indicado para el SCA con SDST, como es la angioplastía primaria $^{25}$. A nivel de biomarcadores de daño miocárdico se observó en todos elevación de troponina en valores medios bajos, coincidente con lo descrito en que el ascenso de troponina en el STT es menor que en $\mathrm{SCA}^{5,6,22}$. Además, se observa en un porcentaje mucho menor de pacientes con ascenso de $\mathrm{CKMB}^{24}$.

En todos nuestros pacientes se planteó el diagnóstico inicial de SCA, como ocurre en la mayoría de los registros ${ }^{3,19,26}$ y se actuó en consecuencia. Algunos (con SDST) fueron a angiografía inmediata, los restantes recibieron tratamiento convencional y fueron a angiografía diferida. La mayor parte sin lesiones coronarias significativas, hallazgos que son similares a los descritos ${ }^{3,5,13,22,26}$ y claramente diferentes al SCA, que en nuestro caso, la mayoría tuvo lesiones coronarias severas. La FE fue menor a lo normal, coincidente con lo publicado ${ }^{5,18,20,22,26}$.

En la evolución hospitalaria, 18,9\% tuvo complicaciones, especialmente falla cardiaca, descrita como la más frecuente ${ }^{18,22,26,27}$. Se ha descrito una variedad de otras complicaciones, también en porcentaje variable, como arritmias de diferentes tipos $^{28}$ e incluso rotura ventricular ${ }^{29}$ con mortalidad hospitalaria desde $0 \%$ a $9 \%{ }^{5,6,8,13,18,22,30,31}$, lo que no permite suponer benignidad del cuadro de STT, como inicialmente se había planteado, 
aunque en las comparaciones con el SCA, este sigue teniendo peores resultados hospitalarios ${ }^{22,32}$, aunque algunos plantean que serian similares ${ }^{5,33}$. En nuestro grupo, la evolución hospitalaria luego de las complicaciones iniciales fue buena. Todos evolucionan hacia la recuperación completa, no registramos mortalidad hospitalaria y luego de un período variable de tiempo todos recuperan la motilidad segmentaria y función ventricular. Esta buena evolución se ha visto en otros registros también ${ }^{8,30,31}$, coincidentemente con números pequeños de pacientes Este hecho podría ser la causa principal de tales buenos resultados. El tratamiento farmacológico indicado al alta fue basado en la intuición, similar a un paciente con SCA, con aspirina, $\beta$-bloqueo, estatinas e IECA, similar a la mayoría de los registros ${ }^{6,18,26,30,31}$. Este tratamiento no tiene ninguna base científica, se discute el real rol del $\beta$-bloqueo, planteándose por algunos que no tiene utilidad ${ }^{5,26}$ y que serían los IECA la mejor alternativa para el largo plazo ${ }^{5}$. Esto aún nos parece que no tiene una respuesta definitiva.

En el seguimiento durante un año no tuvimos mortalidad ni recurrencia del cuadro, los datos disponibles muestran recurrencias entre $0-22 \% 5,6,8,13,18,22,30,31,33$ y mortalidad entre $0-15 \% 5,6,8,13,14,18,22,30,31$, la mayor parte de causa no cardiaca. Nuevamente, algunos llaman la atención de lo grave que puede ser la evolución, incluso similar a un $\mathrm{SCA}^{5,33}$, otros se acercan más a nuestros datos ${ }^{8,18,31,32}$, quizás reflejando poblaciones diferentes o al número reducido de casos que nosotros tenemos aún.

\section{Conclusión}

El STT es de presentación infrecuente. La incidencia encontrada por nosotros levemente inferior a la descrita en la literatura podría explicarse porque solo consideramos la variedad clásica. Simula un SCA, debe ser sospechado por sus particularidades clínicas, electrocardiográficas y enzimáticas. Su confirmación es mediante la coronariografía. La evolución hospitalaria no siempre es benigna. Todos nuestros pacientes normalizan motilidad y función ventricular en el seguimiento, característica diferencial definitiva en comparación con SCA. Este grupo representa la primera serie publicada en América Latina y comparte características comunes con otras series de diferentes lugares del mundo, así como pequeñas diferencias, en especial en mortalidad y recurrencias, diferencias que pueden explicarse por el número de pacientes así como posibles diferencias poblacionales.

\section{Referencias}

1. Dote K, Sato H, Tateishi H, Uchida T, Ishihara M. Myocardial stunning due to simultaneous multivessel coronary spasm: a review of 5 cases. J Cardiol 1991; 21: 203-14.

2. Wittstein I. Stress Cardiomyopathy: A Syndrome of Catecholamine-Mediated Myocardial Stunning? Cell Mol Neurobiol 2012; 32: 847-57.

3. Desmet W, Adriaenssens B, Dens J. Apical ballooning of the left ventricle: first series in white patients. Heart 2003; 89: 1027-31.

4. Ibáñez B, Navarro F, Farré J, Marcos-Alberca P, Orejas M, Rábago R, et al. Asociación del síndrome tako tsubo con la arteria coronaria descendente anterior con extensa distribución por el segmento diafragmático. Rev Esp Cardiol 2004; 57 (3): 209-16.

5. Templin C, Ghadri J, Diekmann J, Napp L, Bataiosu D, Jaguszewski M, et al. Clinical Features and Outcomes of Takotsubo (Stress) Cardiomyopathy. N Engl J Med 2015; 373: 929-38.

6. Kurowski V, Kaiser A, von Hof K, Killermann D, Mayer B, Hartmann F, et al. Apical and midventricular transient left ventricular dysfunction syndrome (takotsubo cardiomyopathy): frequency, mechanisms, and prognosis. Chest 2007; 132: 809-16.

7. Akashi Y, Goldstein D, Barbaro G, Ueyama T. Takotsubo cardiomyopathy: a new form of acute, reversible heart failure. Circulation 2008; 118: 2754-62.

8. Pillière R, Mansencal N, Digne F, Lacombe P, Joseph T, Dubourg O. Prevalence of Tako-Tsubo Syndrome in a Large Urban Agglomeration. Am J Cardiol 2006; 98: 662-5.

9. Ugalde H, Mayor J, Muñoz M, Ugalde D. Síndrome de tako-tsubo: presentación de un caso clínico típico y revisión actualizada del tema. Rev Hosp Clín Univ Chile 2010; 21: 117-23.

10. Rau C, Kauffmann M, Rau C, Cerededa M, Castro G, Massardo T. Síndrome de Tako-Tsubo: caso clínico. Rev Med Chile 2011; 139: 348-52.

11. Prasad A, Lerman A, Rihal C. Apical ballooning syndrome (Tako-Tsubo or stress cardiomyopathy): a mimic of acute myocardial infarction. Am Heart J 2008; 155 : 408-17.

12. Maciel B, Cidrão A, Sousa Í, Ferreira J, Messias Neto V. Pseudo-acute myocardial infarction due to transient 
apical ventricular dysfunction syndrome (Takotsubo syndrome). Rev Bras Ter Intensiva 2013; 25(1): 63-7.

13. Mejía-Rentería H, Núñez-Gil I. Takotsubo syndrome: Advances in the understanding and management of an enigmatic stress cardiomyopathy. World J Cardiol 2016; 8 (7): 413-24.

14. Sharkey S, Windenburg D, Lesser J, Maron M, Hauser $\mathrm{R}$, Lesser J, et al. Natural history and expansive clinical profile of stress (tako-tsubo) cardiomyopathy. J Am Coll Cardiol 2010; 55: 333-41.

15. Schneider B, Athanasiadis A, Stöllberger C, Stollberger C, Pistner W, Schwab J, et al. Gender differences in the manifestation of tako-tsubo cardiomyopathy. Int J Cardiol 2013; 166: 584-8.

16. Stöllberger C, Finsterer J. Why does Takotsubo ("broken heart syndrome") affect more females than males? Int J Cardiol 2011; 147: 175-6.

17. Madias JE. Low prevalence of diabetes mellitus in patients with Takotsubo syndrome: A plausible 'protective' effect with pathophysiologic connotations. Eur Heart J Acute Cardiovasc Care 2016; 5: 164-70.

18. Núñez Gil I, Andrés M, Almendro Delia M, Sionis A, Martín A, Bastante T, et al. Characterization of Tako tsubo Cardiomyopathy in Spain: Results from the RETAKO National Registry. Rev Esp Cardiol (Engl Ed) 2015; 68: 505-12.

19. Sánchez-Jiménez E. Initial clinical presentation of Takotsubo cardiomyopathy with-a focus on electrocardiographic changes: A literature review of cases. World J Cardiol 2013; 5 (7): 228-41.

20. Castillo AM, Ruiz-Bailen M, Aguilar L. Takotsubo cardiomyopathy - a clinical review. Med Sci Monit 2011; 17 (6): RA135-47.

21. Núñez-Gil IJ, Luaces M, García-Rubira JC, Zamorano J. Electrocardiographic criteria in Takotsubo cardiomyopathy and race differences: Asians versus Caucasians. J Am Coll Cardiol 2010; 56: 1433-44.

22. Zalewska M, Bachorzewska H, Tomaszuk A, Drozdowski P, Bychowski J, Krynicki R, et al. Takotsubo cardiomyopathy: serious early complications and twoyear mortality - a 101 case study. Neth Heart J 2016; 24: 511-9.

23. Bybee K, Kara T, Prasad A, Lerman A, Barsness G, Wright $\mathrm{S}$, et al. Systematic Review: Transient Left Ven- tricular Apical Ballooning: A Syndrome That Mimics ST-Segment Elevation Myocardial Infarction. Ann Intern Med 2004; 141: 858-65.

24. Frangieh A, Obeid S, Ghadri JR, Imori Y, D’Ascenzo F, Kovac M, et al; on behalf of the InterTAK Collaborators. ECG Criteria to Differentiate Between Takotsubo (Stress) Cardiomyopathy and Myocardial Infarction. J Am Heart Assoc 2016; 5: 1-13.

25. Steg G, James S, Atar D, Badano L, Blomstrom-Lundqvist C, Borger M, et al. The Task Force on the management of ST-segment elevation acute myocardial infarction of the European Society of Cardiology (ESC). European Heart Journal 2012; 33: 2569-619.

26. Parodi G, Bellandi B, Del Pace S, Barchielli A, Zampini L, Velluzzi S, et al. Natural History of Tako-Tsubo Cardiomyopathy . Chest 2011; 139 (4): 887-92.

27. Vriz O, Brosolo G, Martina S, Pertoldi F, Citro R, Mos L, et al. In-hospital and long-term mortality in Takotsubo cardiomyopathy: a community hospital Experience. Journal of Community Hospital Internal Medicine Perspectives 2016; 6: 1-9.

28. Syed F, Asirvatham S, Francis J. Arrhythmia occurrence with takotsubo cardiomyopathy: a literature review. Europace 2011; 13: 780-8.

29. Aikawa T, Sakakibara M, Takahashi M, Asakawa K, Dannoura Y, Makino T, et al. Critical Takotsubo Cardiomyopathy Complicated by Ventricular Septal Perforation. Intern Med 2015; 54: 37-41.

30. Bybee K, Kara T, Prasad A, Lerman A, Barsness G, Wright S, et al. Systematic Review: Transient Left Ventricular Apical Ballooning: A Syndrome That Mimics ST-Segment Elevation Myocardial Infarction. Ann Intern Med 2004; 141: 858-65.

31. Andrade Ribeiro V, Vasconcelos M, Melão F, Ferreira E, Malangatana G, Maciel J. Short and Long-Term Outcome of Stress-Induced Cardiomyopathy: What Can We Expect? Arq Bras Cardiol 2014; 102 (1): 80-5.

32. Parodi G, Pace S, Charraba N, Salvadori C, Memisha $\mathrm{G}$, Simonetti I, et al. Incidence, Clinical Findings, and Outcome of Women With Left Ventricular Apical Ballooning Síndrome. Am J Cardiol 2007; 99: 182-5.

33. Akashi Y, Nef H, Lyon A. Epidemiology and pathophysiology of Takotsubo syndrome. Nat Rev Cardiol 2015; 12: 387-97. 